\title{
A SPATIAL ANALYSIS OF ROBBERY RATE IN THE CITY OF DETROIT USING EXPLORATORY DATA ANALYSIS APPROACH
}

Esther Akoto Amoako ${ }^{\mathrm{a}, *}$

${ }^{a}$ Spatially Integrated Social Science, Department of Geography and Planning, The University of Toledo, OH, USA,

Esther.Amoako@rockets.utoledo.edu.

Abstract:

Many U.S. cities have experienced rising crime rates in recent years. Crime has inherent geographic quality and tend to concentrate in certain places within the city. To prioritize public safety and crime prevention strategies, it is important to identify where crime is occurring and with what severity. Using spatial statistics including the average nearest neighbour index, Moran's I, Getis-Ord Gi* statistic, and Anselin Cluster and Outlier Analysis, this study investigates robbery locations within the city of Detroit over 5-year period, 2016 to 2020 to identify hot spots, cold spots and spatial patterns across two different spatial scale - block group and census tracts. The study seeks to understand the effect of data aggregation on each spatial scale on the outcome of the analysis to determine the most optimum spatial scale to study robbery rates. The study concludes that, spatial analysis at small scale like block group level is most informative. Policy implications and areas for further research are provided.

Keywords: Robbery, Spatial Analysis, GIS

\section{Introduction}

The Federal Investigation Bureau's (FBI) Uniform Crime Reporting (UCR) program defines robbery as the taking or attempting to take anything of value from the care, custody, or control of a person(s) by force or by threat of force or violence and or by putting the victim in fear. It is important to note that violent crime is a serious offense and it consist of robbery, aggravated assault, nonnegligent manslaughter, and rape (FBI, 2019). These forms of crimes are considered serious offenses because it creates fear and cause damage to victims thereby creating unsafe environment for people. Robbery inspires fear, as it is usually involving unprovoked attack by strangers that include bodily injury or fear thereof (Braga et al., 2011). The threat of robbery victimization has far reaching impact on urban life by influencing individual choices of residents, where to work, and conduct businesses, etc. (Braga et al., 2011). Conversely, reduction in robbery threat in a city may enhance residents' life and wellbeing as crime in general instil fear and create unsafe environment for residents (Liu et al., 2016).

In 2018, the average dollar value of property stolen per reported robbery was $\$ 2,119$ (FBI, 2019). Robberies accounted for an estimated $\$ 598$ million in losses. Residents experienced the highest average dollar loss at $\$ 4,600$ per offense. The FBI has identified robbery as a large city problem. According to the FBI's UCR, there were 78,421 robbery incidents in the United States in 2018 of which majority of them occurred in major metropolitan areas. Crime tends to concentrate in a particular locality as indicated by most empirical criminological studies (Liu et al., 2016; Mitchell, 2011; Chainey \& Ratcliffe, 2005; Clifford \& McKay, 1942).
The cities with the highest violent crime rates tend to share other socioeconomic characteristics including lack of economic opportunities. Most cities on the FBI most dangerous city list have poverty and unemployment rates higher than the national average. Often, the cities are former manufacturing hub clustered in the industrial Midwest. Cities in Indiana, Michigan, and Ohio have all been affected by the decline of American manufacturing and now have the highest rates of crime in the country. Cities like Detroit, Cleveland, and Indianapolis rank on this list. It should be noted that a higher crime rate does not mean the entire city is unsafe. Even within the city, violence is super-local. Law enforcement has long recognized the inherent geographical component of crime by sticking pins into maps displayed on walls where each pin represented crime event (Chainey \& Ratcliffe, 2005).

Mapping and spatial analysis of crime is essential for examining evidence of the diffusion of crime and in evaluating the effectiveness of geographically targeted crime reduction strategy (Reid et al., 2019). Spatial analysis of crime can play important role in the policing and crime reduction process, it can be employed in both an exploratory and more confirmatory manner with the purpose of identifying how ecological factors influence the spatial pattern of crime. Crime mapping can help law enforcement and policy makers to explore crime patterns, offender's mobility, and serial offences over space and time. Identifying crime hot spots and distribution of crime is useful for targeting, deploying, and allocating suitable crime reduction response. The study of robbery incidents in urban area are of great essence for law enforcement to prioritize safety and crime prevention strategies. The main objective of the study is to assess spatial pattern of robbery rates using several spatial 
analysis techniques in the City of Detroit over four-year period (2017 to 2020).

In this study, the null hypothesis is that there is no statistically significant difference between robbery rate and the random pattern. The scope of the study shows spatial distribution of robbery rate in Detroit at the block group and census tracts level. The purpose of the multiscale analysis is to identify the best scale for conducting analysis to obtain insight on robbery rates. Apart from analyzing robbery hot spot and autocorrelation, this study finds the local outliers.

\section{Literature Review}

Crime mapping can play an important role in the policing and crime reduction process, from data collection through to monitoring and evaluation of any targeted response (Chainey \& Ratcliffe, 2005). Geography is basic to policing, and all good police officers are intimately familiar with their beats due to the important role of place in policing and maps are a common tool. Police have long recognized the inherent geographic nature of crime used pin maps to explore crime events. But it was such studies like the Chicago School of 1930s where Shaw and McKay first demonstrated the importance of geography in understanding crime (Shaw \& McKay, 1942).

The role of location in understanding crime trends and other related ecological and demographic variables like unemployment, makes GIS an essential tool in the study of crime due to its ability to capture and analyze nonspatial and geospatial data (ESRI, 2017). In addition, GIS possesses the ability to integrate and manage massive amounts of datasets, making it extremely useful for aggregating crime data, identifying patterns and clusters, and exploring relationship between crime occurrence and various ecological and demographic variables such as income levels, population density, land use types and facilities (American Sentinel University, 2014; ESRI, 2017). Besides displaying where crimes take place, GIS can provide the physical attributes of the places and the environment in which crimes occur (Chamikara, 2014). Maps that display the crime locations or concentrations are useful for directing patrols and other crime prevention resources to where they are most needed. GIS also proven very useful in assessing the effectiveness of crimereduction strategies adopted by agencies as used by the Baltimore County Police (ESRI, 2017).

GIS provides various analytical techniques for analyzing crime including hot spot analysis, regression, and correlation. GIS can also be used to conduct space-time interactions to determine an association between several similarly occurring criminal activities and an offender as well as spatial proximity and diffusion analysis for certain geographic features like schools and apartment complexes.

The use of GIS in crime studies and analysis enables law enforcement agencies to prioritize and allocate scarce resources to areas that are more susceptible to crime (Chamikara, 2014). In a 2013, the Department of Homeland Security, identified GIS was as the backbone of all the department's efforts in monitoring critical infrastructure, identifying areas highly susceptible to crime and understanding crime patterns. While crime analysis using GIS can show crime trends as well as the relationship between crime, location, victim and the offender, GIS has also made it easier to transform and disseminate crime data to residents, who can in turn make informed decision about where to live and work while participating in crime prevention activities within their neighborhoods (Chamikara, 2014). The City of Denver in Colorado for instance has a GIS website with crime information to help residents track crime within their neighborhoods. Mitchell (2011) used GIS to explore the relationship between crime and demographic factors in Pennsylvania. GIS in crime studies has proven to help agencies and individuals better understand crime and respond to problem of criminality in society. It has become a powerful tool in the geographical profiling of offenders, identification of suspicious locations and real time crime response which are necessary for crime reduction and prevention. Many empirical studies have demonstrated the usefulness of GIS in crime studies.

A key issue in analysis of spatial phenomenon such as crime is the choice of appropriate scale for the analysis. For some phenomenon, smaller scale may be appropriate and accurately reveal the underlying spatial pattern which may otherwise be hidden when larger scale is used. Place and crime research has shown that the microlevel analysis account for majority of the variability in spatial pattern of crime (Malleson, 2019). The identification and analysis of crime for smaller area units contribute to improving efficiency and effectiveness of law enforcement (Ackerman \& Murray, 2004). The ability to carefully delineate areas of concentrated criminal activities provide the opportunity to evaluate why crime is occurring in those specific places. If you can explain the pattern, you can accurately predict outcomes and target intervention.

Studies of the spatial patterns of robbery in urban environments have revealed that a small number of micro places generate a disproportionate number of robberies. Certain high-risk facilities, such as streets conners, bars, convenience stores, and banks, at places tend to experience a disproportionate amount of robbery (Braga et al., 2011).

\section{Data and methods}

The data used to measure criminal activities in Detroit were obtained from the City of Detroit Open GIS data Portal. This data reflects reported criminal offenses that have occurred in the City of Detroit. Robbery cases were aggregated for 4-year period (2017-2020) at the census block group and census tract levels. Multi-scale analysis was used to allow for comparison to be made for spatial pattern of robbery rates to identify the optimum scale for crime analysis. Several factors informed the decision to work with 4-year data. First, a cross-sectional study may not have adequate data points (sample size) for meaningful analysis. Second, there were gaps in the data for example, 2014 and 2015 data were missing hence, a 
10-year assessment was not plausible. Finally, the purpose of the study is to provide perspective on recent robbery trends in the City of Detroit and a 4-year analysis will meet this goal. Robbery rate was expressed as the total number of robberies in each area unit divided by the population times 10,000 residents.

\subsection{Methods and analysis}

The study employed four main spatial analysis techniques: Average Nearest Neighbor Analysis, Global Moran's I, Getis-Ord Gi* (Hot Spot Analysis), and Local Moran's I (Anselin Cluster and Outlier Analysis). These techniques are further explained below.

\subsubsection{Average Nearest Neighborhood Distance}

Nearest Neighbor Statistic method was employed to determine if there exist recognizable pattern in robbery cases at all locations within the City of Detroit. The nearest neighbor statistics is the ratio of the observed average distance between nearest neighbors of a point distribution and the expected average nearest neighbor distance (Wong \& Lee, 2005). It is mathematically expressed as: $\mathrm{R}=\mathrm{r}_{\mathrm{obs}} / \mathrm{r}_{\mathrm{exp}}$, where $\mathrm{r}_{\mathrm{obs}}$ is the observed average distance between the nearest neighbors and the $r_{\exp }$ is the expected average nearest neighbors as determined by the theoretical pattern. The expected average distance is determined by theoretical pattern which is uniform or random pattern. To test if the distribution has any recognizable pattern, $\mathrm{R}$ statistic is used for randomness.

The nearest neighbor analysis was introduced by two botanists Clark and Evans (1954) and has been successfully used by geographers to study different phenomenon. The nearest neighbor analysis uses the $\mathrm{R}$ ratio to compare the expected random pattern to the observe pattern to determine the degree of clustering. The $\mathrm{R}$ scale ranges from $\mathrm{R}=0$, indicating complete clustering and $\mathrm{R}=1$ (Random) to $\mathrm{R}=2.149$, indicating completely dispersed pattern (Wong \& Lee, 2005).

\subsubsection{Spatial Autocorrelations (Global Moran's I)}

Moran's I Index is a global measure of spatial autocorrelation based on both the feature locations and feature attributes simultaneously. Given a set of features and associated attributes, it evaluates whether the pattern expressed is clustered, dispersed, or random. The tool calculate the Moran's I index with both a z-score and pvalue. In this study, the Moran's I identify clusters of similar values of robbery rates where they are aggregated at the block group and census tracts level. Moran's, I range from -1 (extremely negative spatial autocorrelation) indicating clustering of dissimilar values to +1 (extremely positive spatial autocorrelation), indicating that similar values are clustered together. This study aims to uncover if robbery rates are clustered at various scale of analysis across the City of Detroit.

\subsubsection{Hot Spot and Cold Spot Analysis (Getis-Ord / Gi*} Statistics)
Although spatial autocorrelation analysis is helpful in detecting clustering of similar values, it cannot tell if those clusters of geographic units contain high numbers of robbery rates (hot spot) or a low number of robbery rates. Therefore, the Getis-Ord Gi* statistic, which is also a global measure of spatial autocorrelation and capable of detecting the presence of hot spots or cold spots over the entire study area was used. It identifies high value polygons neighboring another polygon with high values, or low value polygons neighboring other low value polygons and assigns varying levels of significance. Generating a z-score and p-value for each input feature indicates whether the observed spatial clustering of high or low values will be more pronounced than one would expect in a random pattern distribution of the same values. A polygon feature with high value may not be statistically significant hot spot unless surrounded by other polygon features with high values. For statistically significant positive $\mathrm{z}$-scores, the larger the z-score the more intense the clustering. A z-score near zero indicates no apparent spatial clustering of high values (hot spot), the smaller the $\mathrm{z}$-score, the more intense the clustering of low values (cold spot) (ESRI, 2021). The formular is given as:

$$
\mathrm{Gi}^{*}=n \frac{\sum_{i=1}^{n} w i j x_{j}-\bar{x} \sum_{j=1}^{n} w i j}{\sqrt[s]{n}}
$$

where $x \mathrm{j}$ is the attribute value for feature $\mathrm{j}$, wij is the spatial weight between feature $I$ and $j, n$ is equal to the total number of features and:

$\bar{X}=\frac{\sum_{j=1}^{n} x_{j}}{n}, \mathrm{~S}=\sqrt{\frac{\sum_{j=1}^{n} x_{j}^{2}}{n}}-(\bar{X})^{2}$

\subsubsection{Outlier Analysis (Anselin Local Moran's I)}

All the global measure of spatial autocorrelation, Geary's C and Moran's I and Getis-Ord Gi* provide statistic for the entire study area and assume that the magnitude of spatial autocorrelation is uniform over the study region. This assumption may be misleading as it is likely that the magnitude of spatial autocorrelation is high in some subregions but low in others. Therefore, the local Moran's I (Anselin Cluster and Outlier) analysis technique was applied as the final technique in the study. Local Moran's I help to identify level of spatial autocorrelation at the local scale by deriving value for each spatial unit (Wong \& Lee, 2005). The equation for local Moran's I is given as:

$I_{i}=z_{i} \sum_{j} w_{i j} z_{i j}$,

where $Z_{i}$ and $z_{j}$ are deviations from the mean for the corresponding $x$ values,

$z_{i}=\frac{X_{i}-\bar{X}}{\delta}$

were $\delta$ is the standard deviation of the variable $\mathrm{x} . \mathrm{Z}_{\mathrm{i}}$ is therefore the $\mathrm{Z}$ score of $\mathrm{x}_{\mathrm{i}}$.

The outlier analysis tool calculates a local Moran's I value, a z-score, pseudo $\mathrm{p}$ value, and a code indicating the cluster type for each statistically significant feature. The 
$\mathrm{z}$-score and p-values represent the statistical significance of the computed index value. A positive value for I indicates a feature has neighboring features with similar high or low value. A negative value for I indicates the feature has neighboring features with dissimilar values; these features are outliers. In either case, the $p$ value for the feature must be small for either clusters or outliers to be statistically significant.

\section{Results and Discussions}

\subsection{Descriptive Statistics}

There are 879 block groups and 310 census tracts in Detroit. The City of Detroit Crime Reporting Systems data recorded 8,799 robbery incidents over the course of 4-years (2017 - 2020). Out of the total number of robbery incidents, there were 2,529 incidents in 2017, 2,228 robberies in 2018, 2,266 and 1776 robberies in 2019 and 2020, respectively. Over this period of study, 2020 recorded the lowest robbery cases. The FBI crime statistics shows a general decline in violent crime rate nationwide. For instance, the report revealed $3.3 \%$ decrease in violent crime rate in 2018 from 2017 number. The city of Detroit is also experiencing similar trend of decreasing robbery rates. Figure 1 . shows the total number of robberies per year, which indicates a steady rise in 2017, but a constant decline afterwards.

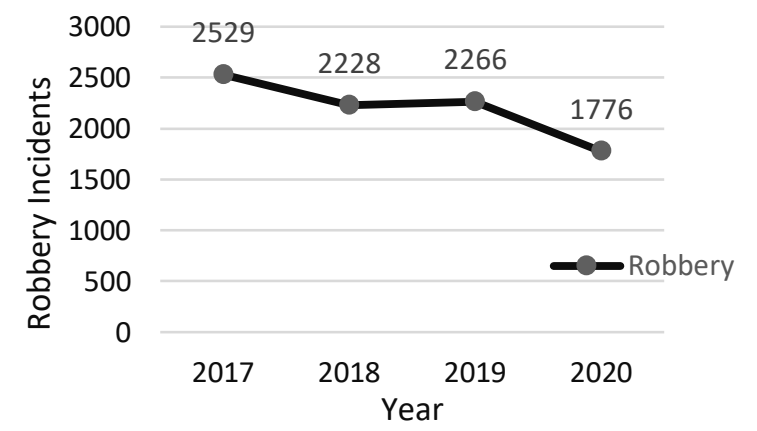

Figure 1. Number of Robbery cases by year in Detroit, Michigan (2017 to 2020)

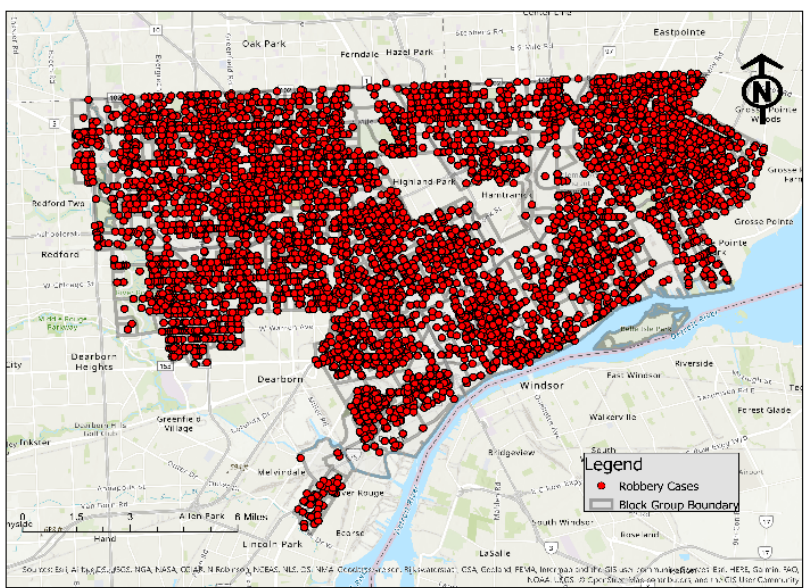

Figure 2. Map of Robbery cases in Detroit, Michigan, 2017 to 2020
Figure 2. shows the distribution of robbery incidents across census block groups in Detroit over the course of five years. A visual clustering of robbery around certain block groups can be seen from the map, especially around the downtown area at the south of the city, eastern and western part of the city. This is to be expected as the downtown area usually have larger share of crime because of the concentration of people and business in the city center.

\subsection{Average Nearest Neighbor Analysis Results}

The result from the average nearest neighbor analysis index (see Table 1) of Detroit robbery incidents produced nearest neighbor index/R ratio of 0.372947 , indicating clustering of robbery points at the city level and statistically significant at a p-value of $<0.000000$. Given the negative $\mathrm{z}$-score of -113.0297 , there is less than $1 \%$ likelihood that this clustered pattern of robbery in Detroit could be the result of random process.

\begin{tabular}{ll}
\hline Robbery Rates at & Block Group \\
Level & \\
Observed (ft) & 165.1076 \\
Expected (ft) & 442.7105 \\
R Ratio & 0.372947 \\
Z-score & -113.0297 \\
P-value & 0.000000 \\
Pattern & Clustered
\end{tabular}

Table 1. Average Nearest Neighbor Analysis Summary for robberies across the city, 2017 to 2020

\subsection{Global Spatial Autocorrelation Results}

To further analyze trends across the city and identify significant clustering points of robbery rates over the 5year period, spatial autocorrelation was computed using the global Moran's I on two different spatial scale; block group and census tracts level (see Table 2).

\begin{tabular}{ll}
\hline Robbery Rates at Block Group & \\
Moran's Index & 0.069081 \\
Expected Index & -0.001139 \\
Z-Score & 5.963265 \\
Variance & 0.000139 \\
P-value & 0.000000 \\
Pattern & clustered \\
Robbery Incidents at Census Tracts \\
Moran's Index & 0.133758 \\
Expected Index & -0.001139 \\
Z-Score & 7.180881 \\
Variance & 0.000353 \\
P-value & 0.000000 \\
Pattern & clustered \\
\hline
\end{tabular}

Table 2. Global Moran's I Summary for Robbery Incidents

The global Moran's I derived for robbery rate at block group level is 0.069081 . Given the z-score of 5.963265, there is a less than 1\% likelihood that this clustered pattern of robbery rates in Detroit block group could be 
the result of random chance. At the census tract level, the Moran's I is 0.133758, indicating positive spatial autocorrelation. This number is also found to be statistically significant. This indicate that robbery rate at the census tracts level follow a clustered pattern. The Moran's I computed for robbery rates at the block group and census tracts were both statistically significant. Thus, there is expected systematic process driving robbery rates in Detroit at both the block group and census tracts level and cannot be a result of random processes. Since, the two different scale of analysis yielded comparable results, the issue relating to the modifiable areal unit problem did not affect the outcome of spatial dependence when the scale of analysis varied. Given the z-score of 9.871029, for census tracts and 5.963265 for block group scale,

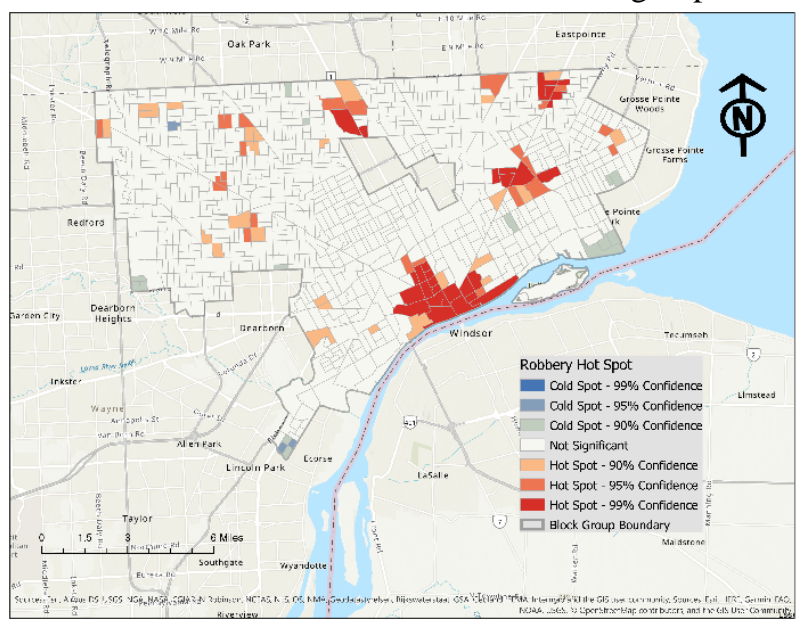

there is a less than $1 \%$ likelihood that this clustered pattern could be the result of random chance and hence we reject the null hypothesis. Given clustering of similar values (positive spatial autocorrelation) as indicated by the Moran's I value at both block group and census tracts level, it is possible similar ecological and sociodemographic factors are driving the pattern of robbery across the city.

\subsection{Hot Spot and Cold Spot Analysis (Getis-Ord Statistic) Results}

While spatial autocorrelation analysis is useful in finding clusters of similar values, it cannot determine if those clusters of geographic units contain a high number of robbery rate (hotspots) or low number of robbery rate

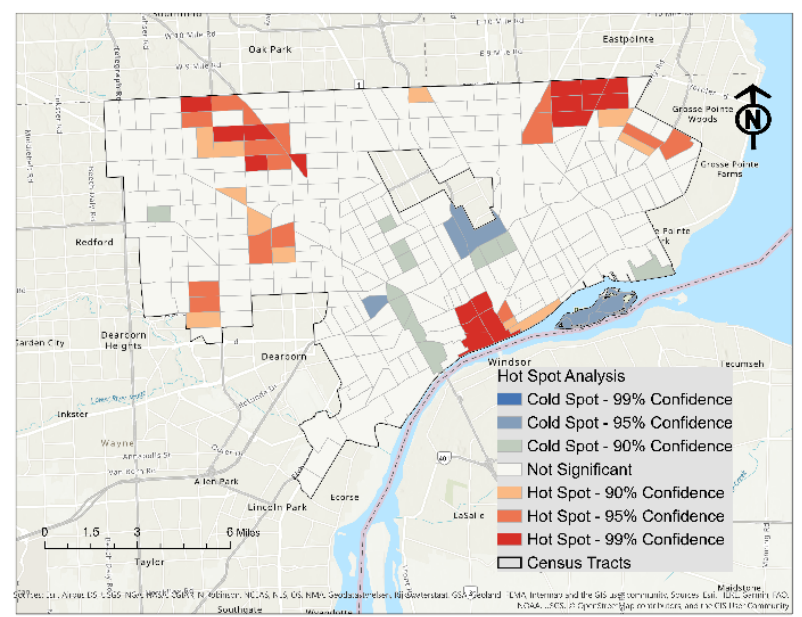

Figure 3. Analysis of Robbery rate by (a) Detroit Block Groups and (b) Census Tracts, 2017-2020

(cold spots). To ascertain this, a hot spot analysis using Getis-Ord Gi* statistic was performed.

Figure 3 a displays the first analysis at the block group level and identified 7 hot spots. All the hot spots are in the South-eastern and Northwest part of the City, major hot spots are found at the downtown area. The Hot spots consist of 31 block groups with $99 \%$ significance level represented by dark red color. These hot spots are surrounded by $95 \%$ significance level hot spot symbolized by the mild shade of red consisting of 25 block groups, which is further surrounded by $90 \%$ significance hot spot (lighter shade of red). The blue colors represent cold spots which indicates areas with low robbery rates that are surrounded by neighbors with low robbery rates. There are few cold spots at the block group level.

Figure $3 \mathrm{~b}$ shows hot spots at the census tract level. Robbery hot spots at the census tracts level shifted towards the Northeast, South and Northwestern part of the City. Significant cold spots are found at the central and on the Island of the City. This shift in pattern can be attributed to modifiable areal unit problem (MAUP) effect arising from changes in the scale of analysis.

The hotspot at the Downtown area is not surprising as this area has many targets for offenders including businesses, restaurants, drinking bars, transportation hub, etc. which tends to attract offenders and is consistent with existing literature (Braga et al., 2011; Liu et al., 2016). The eastern part of the Detroit has high concentration of marginalized population and historically has high concentrated disadvantage including extreme poverty, low median household income, Black and immigrant population, vacant housing etc. These factors may contribute to the pattern we see from the hot spot map.

\subsection{Clusters and Outlier Analysis (Anselin Local Moran's I) Results}

Given a set of weighted features, Anselin Local Moran's I identifies statistically significant hot spot, cold spot, and spatial outliers. Areas of high values surrounded by areas of high values is classified as high-high clusters, low values surrounded by neighbors with low values indicates low-low clusters, and high values surrounded by low values and vice versa indicates outliers.

The analysis at the block group level (Figure 4a) and census tract level (Figure 4b) indicate outliers and clusters, giving much detail to hot spot analysis of robbery rates obtained from Getis-Ord Gi*. Figure 4a shows sporadic high-low (represented by red colors) outliers at the west and central block groups and low high outliers (bright blue color) are sporadic across the study area. The high-high clusters are mainly at the southern part of the city, indicating different pattern from GetisOrd Gi* block group result. High-high clusters indicates 
block groups that has high robbery rate surrounded by block groups with similarly high values of robbery. While High-high and low-low clusters indicates positive spatial autocorrelation, the outliers indicate negative spatial autocorrelation. This means that the areas of outliers have block groups with high robbery rates surrounded by neighbors with low robbery rate and vice versa. It can be seen from the map that the northeastern part of the city has packets of significant clusters of robbery rates as well as the southern part of downtown area. The places on the map showing clusters of high values are surrounded by low-high outliers. This pattern indicates that, the magnitude of robbery at the block group level is not uniform across the study units. The Local Moran's I thus, provides better understanding of the spatial pattern of robbery rate across the city which was not possible with the global indicators of spatial autocorrelation. Unlike the global Moran's I, the local

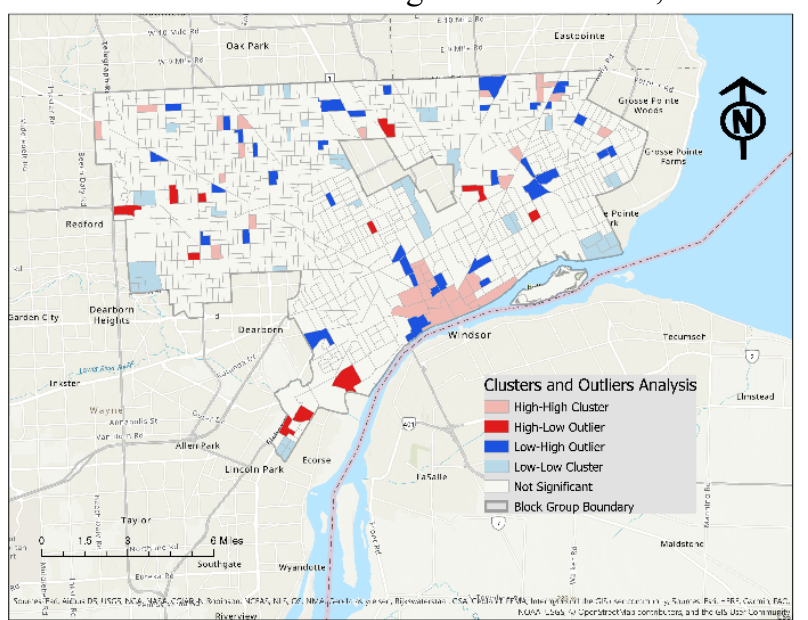

Figure 4. Clusters and Outlier Analysis of Robbery Rate by

\section{Conclusion}

Four spatial analytical techniques were used to evaluate spatial patterns of robbery rates at two different scale in Detroit over a 4-year period. The nearest neighbor analysis at the city level revealed statistically significant clustered pattern of robbery rates, indicating robbery rate in Detroit is driven by systematic processes than random chance. The result from the average neighbor analysis was confirmed by the map showing distribution of robbery rates in the city.

Although the robbery cases have been declining from 2017 to 2020 , robbery is still a major problem within the city and the magnitude of the effects are not uniform across the study region, it tends to cluster in certain block groups and census tracts. The global Moran's I showed clustering of similar values and it was statistically significant at $99 \%$ confidence level. The global Moran's I statistic showed that clustering of robberies at both block group and census tracts level were not a result of some random process but systematic process; leading to rejection of the null hypothesis and the conclusion that robbery rates at census tracts and block group levels are different from random pattern.
Moran's I provides the magnitude of spatial effect at the local level and reveal underlying pattern at the local level which was not possible with the global indicators. For instance, few cold spots were identified by Getis-Ord Gi* statistic at the block group level but the local Moran's I identified all the outliers and high-high/low/low clusters. we can have much confidence with conclusions based on Local Moran's I result than with the Global statistic.

Figure $4 \mathrm{~b}$ shows clusters and outliers at the census tract level. MAUP effect is still evident as the pattern shifted drastically when the scale of analysis changed from block group to census tracts. The high/high cluster of robbery rates at the Downtown area south of the city became smaller than the block group pattern. Additionally, the pattern of robbery rates at the census tracts level are less sporadic than the block group pattern

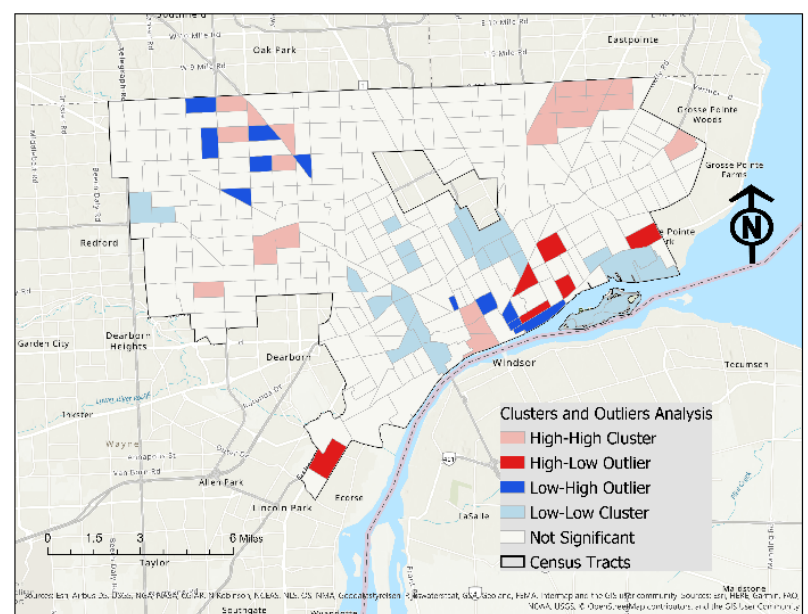

(a) Block Group and Census Tract of Detroit, 2017-2020

Since Moran's I cannot identify where robbery hot spot and cold spot are concentrated, the Getis-Ord Gi* statistic was used. Both block group analysis identified robbery hot spots at the southeast and western part of the city. While census tracts pattern showed pronounced robbery hot spots at the Northeast, Western and Southern part of the city, the block group showed robbery hot spots at the Southeast and sporadic hot spot at the western part of the city. This multiscale level analysis provided much insight into robbery rates which otherwise may not have been possible if a single scale of analysis was used. Again, the multilevel analysis also revealed MAUP effects which reveal useful guide to researchers in drawing study conclusions and inference.

What is common to all the spatial autocorrelation statistics employed (Moran's I and Getis-Ord Gi*) in the study is that they are global statistics which provide summary measure for the entire study region. It is reasonable to suspect that the magnitude of spatial autocorrelation is not necessarily uniform over the study area but varies from one location to another. To better capture variability of autocorrelation for each area unit across the study region, the local Moran's I (Anselin Cluster and Outlier) statistic technique was used. The 
result at both block group and census tracts identified outliers (high-low/low-high) as well as clusters of highhigh values throughout the study region. The eastern part of Detroit had many outliers as clusters of high values. Nonetheless, the outliers were more pronounced at the block group level than at the census tracts scale. The local Moran's I provided much insight on robbery at the local scale which was more informative than what was obtained by the global measures.

The policy implication of the study is that it provides useful information on robbery pattern across the City which can help law enforcement to deploy and allocate suitable crime reduction response to areas much needed. Police and other law enforcement agencies are better equipped with information on robbery hot spots in the city which can help them develop public safety measures to educate the public about where to avoid and exercise the most caution. The result from the study can complement policy effort by the City to support hot spot areas with social intervention resources and programs such as counselling, community revitalization programs, etc. This study could be replicated in other cities experiencing high crime rates such as Baltimore, St. Louis, Chicago, Columbus, among others.

Further studies can focus on exploring the relationship between robbery and ecological and demographic factors to understand the pattern better. Also, this study examined only robbery and hence future studies can look at other forms of violent crime such as homicide, aggravated assault, and rape to see if they follow similar pattern as well as property crime. There is a need for further studies that combines spatial analysis and spatial econometrics models to identify the predictors of crime in the City.

Generalization of the study is only applicable to the study area, and it must be noted that the modifiable unit area problem resulting from scale and zonal effects may influence the pattern shown by the study. The study employed only few spatial statistic methods in the analysis, other methods such as Ripley's K Function, emerging hotspot analysis, etc, may provide useful information. Additionally, the study did not consider neighborhood characteristics that may explain the pattern. Finally, the study only considered robbery rates, other forms of crime such as violent crime, property crime in general or individual crime types were not considered. Besides these limitations, the study provides useful information on robbery situation in the City of Detroit at the block group and census tracts level.

\section{References}

Ackerman, W. V., \& Murray, A. T. (2004). Assessing spatial patterns of crime in Lima, Ohio. Cities, 21(5), 423-437. https://doi.org/10.1016/j.cities.2004.07.008

Andresen, M. A. (2006). A spatial analysis of crime in Vancouver, British Columbia: A synthesis of social disorganization and routine activity theory. The Canadian Geographer / Le Géographe Canadien, 50(4), 487-502.https://doi.org/10.1111/j.1541-

0064.2006.00159.x

American Sentinel University (2014). GIS for Crime Analysis, Law Enforcement and Public Safety. Retrieved from

https://www.americansentinel.edu/blog/wpcontent/uploads/2014/06/AS_GIS-Crime-eBook-

Final.pdf

Block, R. L., \& Block, C. R. (1995). Space, Place and Crime: Hot Spot Areas and Hot Places of LiquorRelated Crime. 39.

Braga, A. A., Hureau, D. M., \& Papa Christos, A. V. (2011). The Relevance of Micro Places to Citywide Robbery Trends: A Longitudinal Analysis of Robbery Incidents at Street Corners and Block Faces in Boston. Journal of Research in Crime and Delinquency, 48(1), 7-32. https://doi.org/10.1177/0022427810384137

Canter, P. R. (no date). Geographic Information Systems and Crime Analysis in Baltimore County, Maryland. Retrievedfrom

https://popcenter.asu.edu/sites/default/files/library/crime prevention/volume_08/06-Canter.pdf

Chainey, S., \& Ratcliffe, J. H. (2005). GIS and Crime Mapping. John Wiley \& Sons, Ltd.

Clifford, R. S., \& McKay, D. H. (1942). The Social Disorganization Theory.

Environmental Systems Research Institute (ESRI). (2021). How Hot Spot Analysis (Getis-Ord Gi*) works. Chamikara, M. A. P. (2014). GIS In Crime Analysis. Sciscitator. 1. 39-40

Department of Homeland Security (2013). Geographic Information System and Predictive Policing Application Note. Retrieved from

https://www.dhs.gov/sites/default/files/publications/GIS-

Predictive-Policing-AppN_0813-508_0.pdf

Emig, M., Heck, R., and Kravitz, M. (1980). Crime

Analysis: A Selected Bibliography. Washington, DC:

US National Criminal Justice Reference Service.

ESRI (2017). Crime Analysis - The Current State. Retrieved from

https://www.esri.com/ /media/Files/Pdfs/library/brochure s/pdfs/gis-for-crime-analysis.pdf

FBI. (2019). FBI Releases 2018 Crime Statistics. FBI National Press Office.

Herrnstein, R. J. and Wilson J. Q. (1985). Are Criminals Made or Born? Retrieved from

https://www.nytimes.com/1985/08/04/magazine/are-

criminals-made-or-born.html

Kennebeck, D. L., \& Zieman, R. W. (2016). Correlation Between Crime and Street Lighting in Rochester, Minnesota USA from 2008-2013. Papers in Resource Analysis, Saint Mary's University of Minnesota University Central Services Press. Winona, MN., 19, 13.

Liu, D., Song, W., \& Xiu, C. (2016). Spatial patterns of violent crimes and neighborhood characteristics in Changchun, China. Australian \& New Zealand Journal of Criminology, 49(1), 53-72.

https://doi.org/10.1177/0004865814547133 
Liu, L., Lan, M., Eck, J. E., \& Kang, E. L. (2020). Assessing the effects of bus stop relocation on street robbery. Computers, Environment and Urban Systems, 80, 101455.

https://doi.org/10.1016/j.compenvurbsys.2019.101455

Malleson, N. (2019). Identifying the appropriate spatial resolution for the analysis of crime patterns. PLOS ONE, 14(6).

Mitchell, S. (2011). Using GIS to Explore the Relationship between Socioeconomic Status and Demographic Variables and Crime in Pittsburgh, Pennsylvania. 11, 11.

O'Connell, H. (2017). What Happens in the Shadows: Streetlights? Rice University Kinder Institute for Urban Research. https://doi.org/10.25611/41qx-u9sm.

Office of the Victims of Crime. (2010). Robbery Victimization. 2010, 2. 